\title{
Modular System for Cold Storage Monitoring
}

\author{
http://dx.doi.org/10.3991/ijoe.v12i04.5127 \\ P.J. Sousa, P. Abreu and M. R. Quintas \\ University of Porto, Porto, Portugal
}

\begin{abstract}
This work describes the development of an embedded electronic-based monitoring system suitable for cold-storage electrical equipment. The system uses a touchscreen and provides sensors for temperature, relative humidity, electric power consumption and detection of door position. To monitor the electric power, a special purpose current sensor was developed and calibrated. The system adopts a modular architecture using cabled and wireless communications, making it suitable for integration in other logging and alarm generation systems. The system was tested on a home fridge to demonstrate its capabilities.
\end{abstract}

Index Terms-Electric Power Measurement, Monitoring System, Relative Humidity, Temperature.

\section{INTRODUCTION}

Currently, a growing number of companies and research institutions rely on technological equipment for their daily activity. Therefore, it's important to constantly monitor the performance of critical equipment using external monitoring systems. For the food and pharmaceutical industries, cold storages, used for maintaining products and product samples [1-3] are an example of equipment to be considered.

The main goal of this work was to develop a low cost cold storage monitoring system for data logging based on microcontrollers and embedded electronics. The temperature, the relative humidity, the access door state and the electrical power consumption are the parameters chosen to be monitored. Among the features that a monitoring system must comply with is the temperature accuracy class that classifies the temperature recording systems. The developed system is compliant with the Accuracy Class 1 defined by the ISO 12830 standard: Maximum Error of $\pm 1^{\circ} \mathrm{C}$ and Resolution $\leq 0.5^{\circ} \mathrm{C}$ [4].

\section{MONITORING SYSTEM}

The developed system [5] provides sensing, data logging and visualization functionality. It has a modular configuration using cabled and wireless communications providing each module with independent and autonomous functions. The system architecture, Fig. 1, makes use of three different modules:

- The central module acts as a Human-Machine Interface (HMI) through the use of a tactile color display and collects all the information from the other modules. It is powered by a 9V DC transformer and manages the USB storage and the communication;

- The electric power metering module uses a current sensor specially built and is connected to the central module through a wireless connection using the MiWi protocol. This module measures the

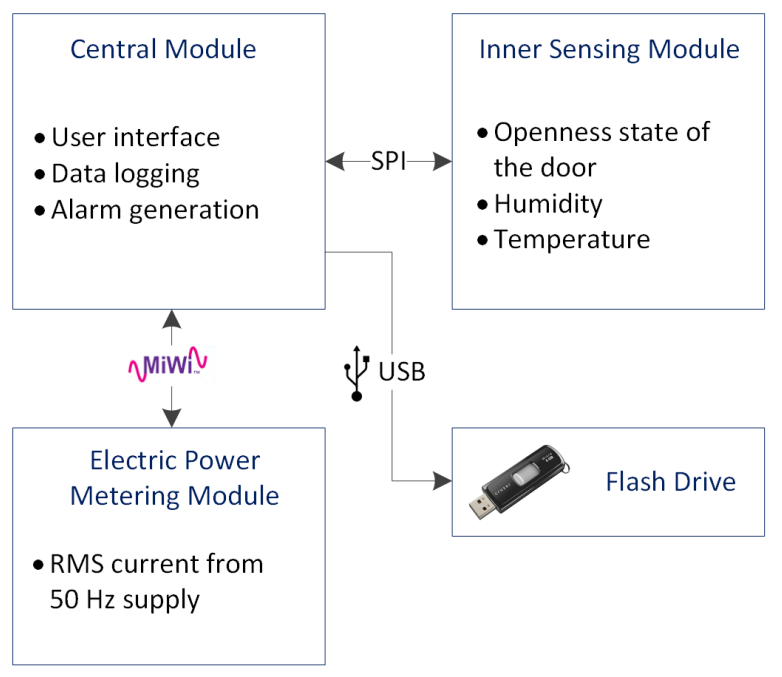

Figure 1. Architecture of the developed system

power consumption of the cold storage and is powered directly from the mains supply;

- The inner sensing module is powered by a wired connection to the central module, through which it transmits relevant data using the Serial Peripheral Interface (SPI) protocol. This module supplies temperature, relative humidity and access door state to the central module.

Each module is autonomous, allowing it to be easily integrated in different products for a wide variety of application areas. A future expansion of this system can be easily accomplished, either integrating new sensors or entirely new modules. The central module was used by $[6$, 7] as the Rehabilitation Glove's HMI.

\section{A. Central Module}

This module comprises two microcontrollers, one responsible for MiWi wireless communication and the other responsible for the remaining interfaces. They communicate through the Serial Peripheral Interface (SPI) protocol and exchange data at a rate of $1 \mathrm{~Hz}$.

The first microcontroller makes use of Microchip's MLA (Microchip Library for Applications) to manage both the Touchscreen, through its SSD1926 controller and a resistive touch panel, and the USB Host mode communications, through an hardware module inside the microcontroller. The communication protocol required by the humidity sensor, 1-wire, was implemented using specifically developed software.

The second microcontroller makes use of the same library, MLA, for MiWi wireless communications and connects to an MRF24J40MA antenna. 
This module's housing was designed and 3D printed inhouse and is capable of supporting the main circuit board as well as potential add-on boards. The finished prototype of the Central Module is presented in Fig. 2.

\section{B. Inner Sensing Module}

This module measures Humidity and Temperature inside the cold storage and detects the openness state of the access door. It is connected to the central module through an RJ45 cable that was selected because of its availability and the clipping functionality it provides. Communications are based on the Serial Peripheral Interface protocol.

It features a DHT22 temperature and relative humidity sensor, a type $\mathrm{K}$ thermocouple and a Hall-effect sensor. This allows for redundancy in temperature readings, which allows the system to choose an alternative reading in the case of a problem with one of the sensors.

To allow better readings from the thermocouple, a regulator with insulation, Texas Instruments' DCR010503, was used.

This module's housing was also designed and 3D printed in-house and features rubber insulation to allow it to better withstand the conditions inside the fridge. Fig. 3 shows the finished Inner Sensor Module.

\section{Electric Power Metering Module}

This module measures the electric current consumption of the cold storage. It was designed to provide a sampling rate of $1 \mathrm{kHz}$, that is, 20 times greater that the frequency of the AC signal that is being measured, $50 \mathrm{~Hz}$. This allows for an adequate description of the source signal [8]. The current data is sent to the central module using the wireless communication protocol MiWi. This allows the module to be placed between the power plug and the wall socket.

It is powered by the current it measures to avoid unnecessary power supply connections and contains a custom current sensor. The sensor uses a coil and a Hall Effect sensor that measures the induced current. The output voltage of the Hall sensor is proportional to the current flowing in the coil. The number of turns of the coil and the range of the current to be measured affects the resolution and sensibility of sensor output. The sensor was customized for reading AC RMS currents up to 2.5 A and tested. The results revealed a characteristic with high linearity, $\mathrm{R}^{2}=0.9976$, as can be seen in Fig. 4. This characteristic was obtained using an ammeter to measure the output current of a variable autotransformer connected to an $\mathrm{AC}$ motor.

The housing that was used for this module was from an old electronic On-Off switch to avoid the need to design and $3 \mathrm{D}$ print a functional power plug. The completed prototype of the Electric Power Metering Module is shown in Fig. 5.

\section{Human-Machine Interaction}

The prototype of this monitoring system can generate and register different types of alarms, from connection problems to user-defined temperature trigger values. The alarm management system can be easily configured through the Graphical User Interface.

It also features data logging to an USB flash drive with two separate logs: a log of the measured values in the form of a csv format file for each day and a $\log$ of the generated alarms in a single. $\log$ file.

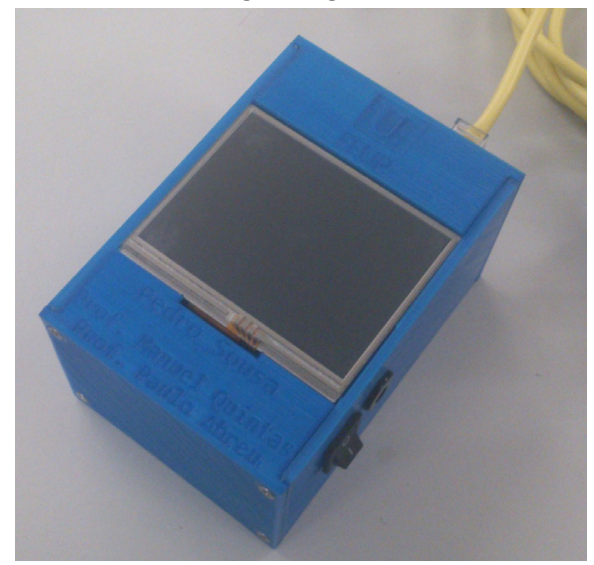

Figure 2. Prototype of the Central Module

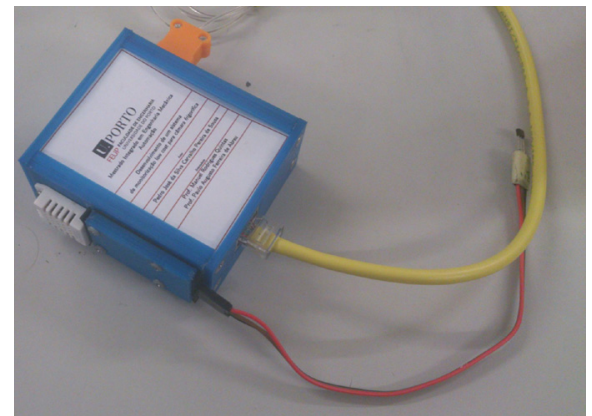

Figure 3. Prototype of the Inner Sensor Module

$A C$ characterization of the developed sensor

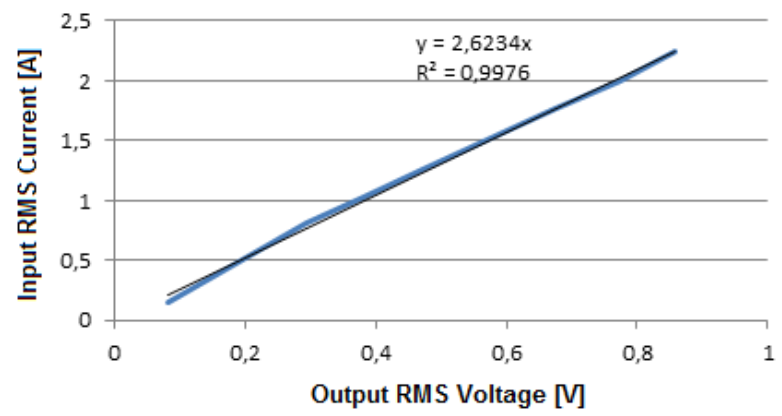

Figure 4. AC characterization of the developed electrical current sensor

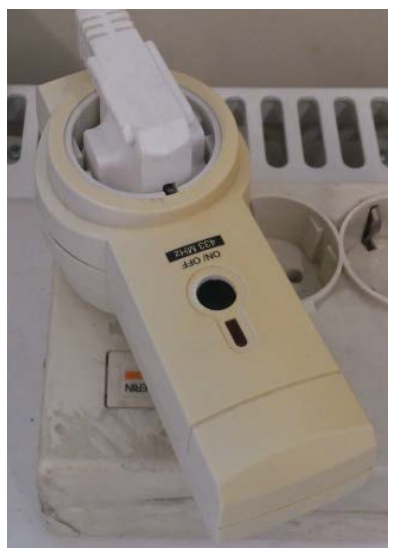

Figure 5. Prototype of the Energy Power Metering Module 
The developed graphical user interface, based on the Microchip Graphics Library, was developed using the Graphics Display Designer X (GDDX) and allows the user to:

- Activate and deactivate the USB data logging;

- View the acquired data: either numerically or through live strip charts like the temperature chart shown in Fig. 7;

- Acknowledge the alarms that were generated;

- Configure the system: define the temperature trigger values that generate alarms, set the RealTime Clock and Calendar (RTCC), among others.

\section{E. Possible Applications}

This monitoring system was developed with cold storages in mind, that is, to monitor the storage conditions of perishable products, such as food, biologic samples or medicines. However, it can be used for several other applications, such as:

- Monitoring controlled experimental environments;

- Evaluating the air quality in confined spaces;

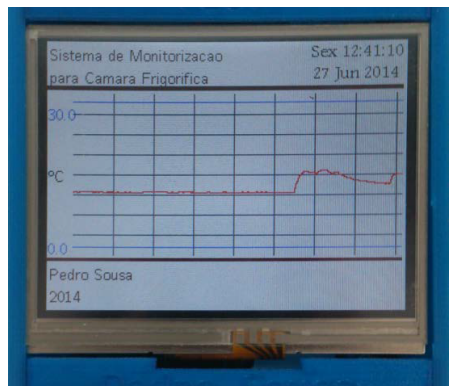

Figure 7. Graphical User Interface: Temperature

- Measuring a device's electric power consumption;

- Monitoring the conditions in greenhouses.

Since it already includes several communication protocols and each module is autonomous, it can be used as a building block to configure different systems.

\section{RESULTS}

A prototype was built for each module of the system and they were assembled on a home fridge.

To test the system, the fridge's door was opened for a two minutes in order to induce significant variations to the thermodynamic conditions inside it. Afterwards, the fridge was allowed to remain with its door closed. Having logged this entire process, the data was exported to the USB flash drive in CSV format. This data was plotted (Fig. 6) using MATLAB. An analysis of the data provides information about the moment the door was open, the time it took the compressor to turn on and the temperature and humidity time behavior. As it can be seen in Fig. 6, the temperature and the relative humidity rose while the door was open, even though the fridge's control system turned on the compressor driving motor. The power consumption complies with the fridge's manufacturer's data.

\section{CONCLUSION}

It was possible to develop a monitoring system for cold storages based on microcontrollers and embedded electronics, while maintaining costs under $€ 250$ for the prototype.

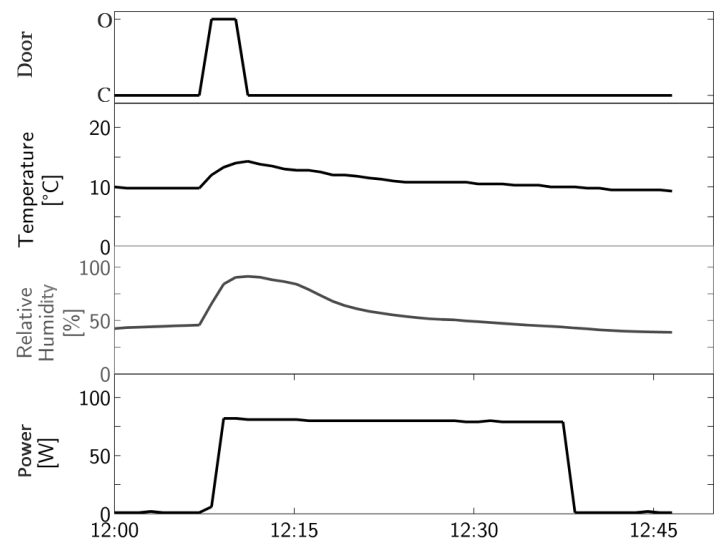

Figure 6. Plotted graph of the data obtained during a test

This system implements a wide range of communications, both wired and wireless, associated with a software application that illustrates the possibilities granted by the developed modules.

The main features of this system are its versatility and autonomy, allowing several uses not only for the system as a whole but also for each of its individual modules.

\section{REFERENCES}

[1] Ü. Kartoğlu, E. Nelaj, and D. Maire, "Improving temperature monitoring in the vaccine cold chain at the periphery: An intervention study using a 30-day electronic refrigerator temperature logger (Fridge-tag $\left.{ }^{\circledR}\right), "$ Vaccine, vol. 28, pp. 40654072, 5/28/2010.

[2] Sistemas de gestão da segurança alimentar. Requisitos para qualquer organização que opere na cadeia alimentar NP EN ISO 22000:2005, Instituto Português da Qualidade ISO 22000.

[3] J. Taylor, "Recommendations on the control and monitoring of storage and transportation temperatures of medicinal products," Pharma. J., vol. 267, pp. 128-131, 2001.

[4] Registadores de temperatura para transporte armazenagem e distribuição de alimentos refrigerados, congelados, ultracongelados e cremes gelados ensaios, avaliação de desempenho, aptidão NP EN 12830: 2008, Instituto Português da Qualidade ISO 12830 .

[5] P. J. Sousa, M. Quintas and P. Abreu, "Monitoring system for cold storages", in $3^{\text {rd }}$ Experiment@ International Conference, Ponta Delgada, Portugal, 2015, pp. 139-140.

[6] R. Tavares, "Desenvolvimento de um dispositivo passivo para reabilitação motora de uma mão," Master's thesis, Faculdade de Engenharia da Universidade do Porto, Porto, 2014.

[7] R. Tavares, M. Quintas and P. Abreu, "Instrumented Glove for Rehabilitation Exercises", in $3^{\text {rd }}$ Experiment@ International Conference, Ponta Delgada, Portugal, 2015, pp. 107-108.

[8] B. G. Liptak, Instrument Engineers' Handbook, Fourth Edition, Volume Two: Process Control and Optimization: CRC Press, 2005 .

\section{AUTHORS}

P. J. Sousa is with the INEGI research center, University of Porto, Porto, Portugal (psousa@inegi.up.pt).

M. R. Quintas and P. Abreu are with the UISPALAETA/INEGI research center and with Faculty of Engineering of University of Porto, Porto, Portugal (mrq@fe.up.pt,pabreu@fe.up.pt).

This work was funded by the National Science Foundation (FCT Portuguese Foundation for Science and Technology) under the project UID/EMS/50022/2013. Submitted 09 October 2015. Published as resubmitted by the authors 23 February 2016. 